\title{
Measurement of single electron spin with sub-micron Hall magnetometer
}

\author{
Jinshuang Jin and Xin-Qi Li \\ Institute of Semiconductors, Chinese Academy of Sciences, P.O. Box 912, Beijing 100083, China
}

(Dated: May 2, 2019; Ms.\# L04-5566)

\begin{abstract}
Sub-micron Hall magnetometry has been demonstrated as an efficient technique to probe extremely weak magnetic fields. In this letter, we analyze the possibility of employing it to detect single electron spin. Signal strength and readout time are estimated and discussed with respect to a number of practical issues.
\end{abstract}

PACS numbers: 06.30.-k,72.10.-d,76.20.+q

Hall probes have been employed in studies of magnetic properties of materials for several decades. Based on the transport properties of quasi-one-dimensional (Q1D) Hall bar system under non-uniform magnetic field [1], the Hall technique has been extended to sub-micron probes for individual microfabricated samples [2]. It was found in several regimes the Hall magnetometer has advantages over the alternative techniques such as $\mu$-SQUIDs [3] and $\mu$-mechanical cantilevers [4].

In recent years, largely being stimulated by the interest of solid-state quantum computation, measurement of single electron spin is becoming an intensive research subject. In particular, it has been suggested that the technique based on the magnetic resonance force microscopy (MRFM), i.e., the $\mu$-mechanical cantilever technique, is probably one of the most promising means [5]. In concern with the sensitivity of the micro-Hall magnetometer, it has allowed to register magnetic changes of $10^{3} \sim 10^{5} \mu_{\mathrm{B}}$ in the sensitive area of cross junction ( $\mu_{\mathrm{B}}$ is the Bohr magneton) [2], which is similar to the demonstrated sensitivity of MRFM technique. Remarkably, recent progress on the MRFM is seemingly to allow the possibility to register single electron spin [6]. In this letter, we address the issue to detect single electron spin based on the sub-micron Hall magnetometer.

The measurement setup is shown schematically in Fig. 1 , where the magnetic dipole (electron spin) to be measured is placed at $z_{0}$ above the cross center of the Hall magnetometer. Due to the presence of the magnetic dipole, non-zero magnetic field exists in the cross region of the Hall junction. The Hall-meter senses this magnetic field by relating it with the Hall signal (Hall resistance or Hall voltage). Conceptually, the Hall signal depends on the dipole (spin-up or spin-down) state, which in physical principle is a counterpart of the well-studied problem of charge qubit (quantum bit) measured by quantum-pointcontact (QPC) [7] or single-electron-transistor (SET) [8].

For simplicity, we assume four identical leads which are fabricated from the two-dimensional electron gas (2DEG), each having a width $W$ and connecting to an electron reservoir with chemical potential $\mu_{i}$. In the linear response regime, from the Landauer-Büttiker formula [9], the Hall resistance can be expressed in terms of the

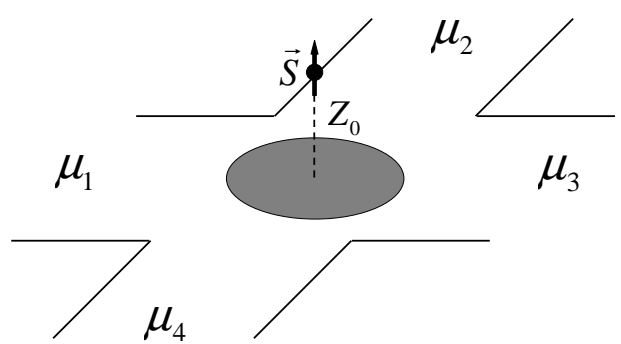

FIG. 1: Schematic setup for the sub-micron Hall magnetometer employed to detect single electron spin.

transmission probabilities $T_{i j}$ (from lead " $j$ " to lead " $i$ ")

$$
R_{H}=\frac{\left(\mu_{2}-\mu_{4}\right) / e}{I}=\frac{h}{2 e^{2}} \frac{T_{21}-T_{41}}{Z},
$$

where $Z=T_{21}^{2}+T_{41}^{2}+2 T_{31}\left(T_{31}+T_{21}+T_{41}\right)$. Here, the current boundary condition $I_{1}=-I_{3}=I$ and $I_{2}=I_{4}=$ 0 are adopted for the Hall measurement.

In this work, we focus on the extremely low temperature which is required for any quantum measurement. In this regime, the electron motion in the Hall bar is ballistic, and a semi-classical Monte Carlo simulation is applicable to compute the transmission probabilities $T_{i j}$ 1]. This approach can easily handle arbitrary inhomogeneous magnetic-field profiles in the Hall cross. It was found that in the regime of low magnetic field, the Hall resistance is determined by the average magnetic field in the cross junction, and is independent of the shape and position of the profile in the junction. In particular, in the weak magnetic field regime a universal relation was found between the Hall resistance and the average magnetic field $\langle B\rangle$ in the junction region [1]:

$$
R_{H}=\frac{\langle B\rangle}{2} \frac{R_{0}}{B_{0}}
$$

where $R_{0}=\left(h / 2 e^{2}\right) \pi / k_{F} W$, and $B_{0}=m v_{F} / e W$. The Fermi wave-vector and velocity is related with the Fermi energy via $E_{F}=\hbar^{2} k_{F}^{2} / 2 m=m v_{F}^{2} / 2$, where $m$ is the effective electron mass of the 2DEG.

Combining Eqs. (11) and (2), we are able to relate the Hall voltage with the parameters of the Hall magnetome- 


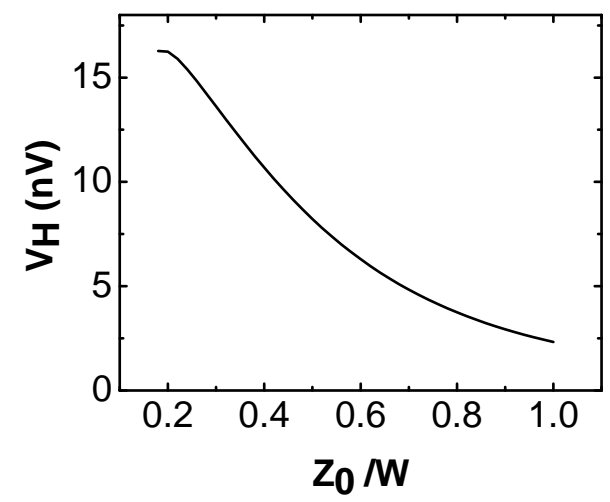

Fig. 2 by Jin and $\mathrm{Li}$

FIG. 2: Hall voltage versus the distance $z_{0}$ where the electron spin is placed above the cross center. Other parameters are referred to the main text.

ter. First, we obtain

$$
T_{21}-T_{41}=\frac{Z \pi \hbar e}{4 m} \frac{\langle B\rangle}{E_{F}} \equiv C_{1} \frac{\langle B\rangle}{E_{F}} .
$$

In the regime of (extremely) weak magnetic field as considered in this letter, " $Z$ " can be well approximated to be a constant independent of the magnetic field, thus " $C_{1}$ " is a constant. On the other hand, based on the Landauer-Büttiker formula [9], it is straightforward to derive $V_{H}=\left[\left(T_{21}-T_{41}\right) / C_{2}\right] V_{13}$, where $V_{13}=\left(\mu_{1}-\mu_{3}\right) / e$ is the voltage applied across the leads "1" and " 3 ", and $C_{2} \equiv T_{21}+T_{41}+2 T_{31}$ is also a magnetic-field independent constant in the weak magnetic field regime. Accordingly,

we arrive at a simple expression for the Hall voltage

$$
V_{H}=\frac{C_{1}}{C_{2}} \frac{\langle B\rangle}{E_{F}} V_{13}
$$

This result clearly shows that the Hall signal (voltage) is determined by the average magnetic field $\langle B\rangle$ and the Fermi energy $E_{F}$ of the confined 2DEG. Note that $\langle B\rangle=\Phi / W^{2}$, where $\Phi$ is the magnetic flux penetrating through the cross region. For the single electron spin measured by the Hall magnetometer, the magnetic flux is distributed in a limited range of nanometers in the Hall cross. We then suggest that the Hall junction should be designed with narrow width " $W$ " in order to enhance the Hall signal. Also, for the same purpose, small Fermi energy $E_{F}$ is favorable, which can be achieved by doping relatively low density of electron numbers as the 2DEG.

To carry out a quantitative estimate, we consider the 2DEG formed from the GaAs heterostructure, which has an effective electron mass of $m=0.067 m_{e}$. Other parameters assumed are the equilibrium Fermi energy $E_{F}=10$ meV which corresponds to electron-number density of $n_{e}=2.8 \times 10^{11} \mathrm{~cm}^{-2}$, and the junction channel width $W=10 \mathrm{~nm}$. To estimate the average magnetic field in the cross region, consider $z_{0}=5 \mathrm{~nm}$ at which the electron is located above the cross center, giving rise to $\langle B\rangle \simeq 8.5 \times 10^{-2} G$. Using these parameters, we can easily compute the four relevant transmission probabilities $T_{j 1}(j=1, \ldots, 4)$, based on Eq. (3) and (i) the probability conservation condition $\sum_{j} T_{j 1}=1$, and (ii) the values of $T_{11} \simeq 2.5 \times 10^{-4}$ and $T_{31} \simeq 0.41$, which are obtained from direct numerical simulations. We thus arrive at an estimate for the Hall voltage, $V_{H} \simeq 10^{2} \mathrm{e} V_{13} / E_{F}$ (in units of nano-volts). As an example, let us set the ratio $e V_{13} / E_{F}=0.1$, which leads to a Hall voltage of $\sim 10$ $\mathrm{nV}$. The result for a wider range of parameter is shown in Fig. 2, where the dependence of $V_{H}$ on $z_{0} / W$ is plotted (with other parameters unchanged as above). We notice that the Hall signal about $10 \mathrm{nV}$ will be within the access of modern technology of voltage measurement, provided other noisy fluctuations can be well suppressed.

In addition to the above estimate of signal strength, below we address the issue of quantum measurement, by regarding the spin as a quantum bit (qubit). Suppose that the spin is subject to an operation (i.e. undergoing Rabi oscillation). We then consider the influence of the Hall measurement on it. The spin Hamiltonian reads $H_{S}=\epsilon_{\uparrow}|\uparrow\rangle\left\langle\uparrow\left|+\epsilon_{\downarrow}\right| \downarrow\right\rangle\langle\downarrow|+\Omega(\mid \downarrow$ \rangle$\langle\uparrow|+| \uparrow\rangle\langle\downarrow|)$. Correspondingly, the electron transport in the Hall magnetometer is governed by $H_{D}=$ $H_{R}+H_{T}$. Here $H_{R}=\sum_{m=1}^{4} \sum_{k} \epsilon_{m k} c_{m k}^{\dagger} c_{m k}$ is the Hamiltonian of the four electron reservoirs, and the tunneling Hamiltonian reads $H_{T}=\sum_{m, n}\left(t_{m n}|\downarrow\rangle\langle\downarrow\right.$ $\left.\left.\left|+\tilde{t}_{m n}\right| \uparrow\right\rangle\langle\uparrow|\right)\left[\sum_{k q}\left(c_{m k}^{\dagger} c_{n q}+\right.\right.$ H.c. $\left.)\right]$, where the summation is over all the transport channels " $(m, n)=$ $(1,2),(1,3),(1,4),(2,3),(2,4),(3,4) "$. Alternatively, let us re-express $H_{T}$ as $H_{T}=\sum_{\alpha=\uparrow, \downarrow} Q_{\alpha} F_{\alpha}$, where $Q_{\uparrow}=$ $|\uparrow\rangle\left\langle\uparrow\left|, Q_{\downarrow}=\right| \downarrow\right\rangle\langle\downarrow|$, and the corresponding $F_{\alpha}$ can be accordingly determined. In this form, the measurement device is clearly playing a role of dissipative environment, and the measured spin would suffer dephasing and relaxation owing to the back-action of measurement. Precisely following Ref. 10 , the $T_{1}$-relaxation and $T_{2}$-dephasing rates read

$$
\begin{aligned}
\frac{1}{T_{1}}= & \frac{\sin ^{2} \theta}{4}\left\{\chi^{2} \sum_{m n}{ }^{\prime}\left[F\left(e V_{m n}+\Delta\right)+F\left(e V_{m n}-\Delta\right)\right]\right. \\
& \left.+T_{24}\left[F\left(e V_{H}+\Delta\right)+F\left(e V_{H}-\Delta\right)\right]\right\} \\
\frac{1}{T_{2}}= & \frac{1}{2 T_{1}}+\frac{\cos ^{2} \theta}{2}\left[\chi^{2} \sum_{m n}{ }^{\prime} F\left(e V_{m n}\right)+T_{24} F\left(e V_{H}\right)\right](5)
\end{aligned}
$$

Here $\chi=\left|\sqrt{T_{41}}-\sqrt{T_{21}}\right|$, and $F(x)=x \operatorname{coth}(\beta x / 2)$, with $\beta$ the inverse temperature. The mixing angle " $\theta$ " is introduced by $\cos \theta=2 \epsilon / \Delta$, or $\sin \theta=2 \Omega / \Delta$. $\epsilon$ and $\Delta$ are, respectively, the spin-up and spin-down level offset and the eigen-energy difference, defined by $\epsilon=\left(\epsilon_{\uparrow}-\epsilon_{\downarrow}\right) / 2, E_{0}=$ $-\sqrt{\epsilon^{2}+\Omega^{2}} \equiv-\Delta / 2$ and $E_{1}=\sqrt{\epsilon^{2}+\Omega^{2}} \equiv \Delta / 2$, where $\left(\epsilon_{\uparrow}+\epsilon_{\downarrow}\right) / 2$ has been taken as the reference energy (i.e. energy zero). $V_{m n}$ is the voltage between the $m$ th and $n$th reservoirs, and the partial summation $\sum_{m n}^{\prime}$ is over 
transport channels " $(m, n)=(1,2),(1,4),(2,3),(3,4)$ ". In our treatment, the different back-action of electron tunneling in channels " $(1,3)$ " and " $(2,4)$ " on the spin has been taken into account, i.e., the former is independent of the spin state, thus has no dissipative effect on the spin dynamics, while the latter affects the spin significantly, because of the opposite Hall voltages for different spin states.

Based on Eq. (5) and the numerical estimate obtained above, we find $T_{1}$ and $T_{2}$ to be about $\sim 10^{-6}$ sec. In general, the quantum measurement time $\left(t_{\text {meas }}\right)$ is in between $T_{1}$ and $T_{2}$ [8]. As a consequence, if the spin coherence time, which is limited by other scattering mechanisms, is longer than the measurement time, the proposed Hall magnetometer would enable not only classical measurement as already discussed above, but also quantum measurement. To perform quantum projective measurement, this readout-time analysis is necessary. However, to our knowledge, similar analysis/estimate of the measurement time based on realistic device setup is widely lacking in the recently proposed quantum measurement schemes [5, 7, 8].

Finally, a number of practical issues/difficulties are remarked as follows: (i) We have restricted our analysis in the ballistic regime which makes sense for narrow Hall junctions and/or for a high mobility 2DEG (e.g. at very low temperature). For completeness, here we compare it with the qualitative result in the diffusive regime which is valid under the opposite conditions. In this case, $R_{H} / R_{0} \simeq\langle B\rangle \mu$, with $\mu$ the $2 \mathrm{DEG}$ mobility [11. Similar derivation as in the ballistic regime leads to $V_{H} /\left(\langle B\rangle V_{13}\right)=\gamma \mu$, with $\gamma$ in the order of magnitude of unity. For the n-type GaAs 2DEG, taking the typical value $\mu=8.35 \times 10^{3} \mathrm{~cm}^{2} / \mathrm{V}$ sec at room temperature [11, we find $V_{H} /\left(\langle B\rangle V_{13}\right) \simeq 0.83 \mathrm{Tesla}^{-1}$, which is sim- ilar to the ballistic result $V_{H} /\left(\langle B\rangle V_{13}\right)=C_{1} /\left(C_{2} E_{F}\right) \simeq$ $0.96 \mathrm{Tesla}^{-1}$. Obviously, with decreasing temperature the diffusive formula would break down, and the Hall voltage does not linearly depend on the increasing mobility. In this situation, the ballistic formula should be adopted. (ii) At finite temperature, the sensitivity of the Hall magnetometer would be fundamentally limited by the (thermal) Johnson noise $V_{H}=\sqrt{4 k_{B} T R_{s} f}$, where $R_{s}$ and $f$ are the series resistance of the device and the measurement bandwidth 12, 13. At $T=4.2 \mathrm{~K}$, the typical series resistance $R_{s}=1.5 \mathrm{~K} \Omega$, and the measurement bandwidth $f=1 \mathrm{KHz}$. We then arrive at a voltage noise about $6 \mathrm{nV}$, which approximately reaches the detection limit (i.e. signal equals noise). In practice, this thermal noise may be suppressed further by decreasing temperature or improving the measurement techniques. (iii) It would be difficult to fabricate the assumed $10 \mathrm{~nm}$ conducting channels. The difficulty originates from the side wall depletion effect. Viewing the depletion layer of $\sim 100 \mathrm{~nm}$ at each side wall, controlling a width of $10 \mathrm{~nm}$ conducting channel would be a challenging job.

In summary, our analysis shows that under appropriate setup design the sub-micron Hall magnetometer can generate a Hall signal of $\sim 10$ nano-volts when measuring a single electron spin. We thus anticipate that if the thermal-noise suppression and fabrication difficulty can be well resolved, the proposed Hall device is a promising candidate of single electron spin sensor.

Acknowledgments. Support from the National Natural Science Foundation of China (NNSFC), and the Major State Basic Research Project No. G001CB3095 of China are gratefully acknowledged.
[1] X.Q. Li, F.M. Peeters, and A.K. Geim, J. Phys.: Condens. Matter 9, 8065 (1997); F.M. Peeters and X.Q. Li, Appl. Phys. Lett. 72, 572 (1998).

[2] A.K. Geim, I.V. Grigorieva, J.G.S. Lok, J.C. Maan, S.V. Dubonos, X.Q. Li, F.M. Peeter, and Yu.V. Nazarov, Superlatt. Microstruct. 23, 151 (1998); A.K. Geim, S.V. Dubonos, J.G.S. Lok, I.V. Grigorieva, J.C. Maan, L.T. Hansen, and P.E. Lindelof, Appl. Phys. Lett. 71, 2379 (1997); A.K. Geim, I.V. Grigorieva, S.V. Dubonos, J.G.S. Lok, J.C. Maan, A.E. Filippov, and F.M. Peeters, Nature (London) 390, 259 (1997); A.K. Geim, S.V. Dubonos, JJ Palacios, I.V. Grigorieva, M. Henini, JJ Schermer, Phys. Rev. Lett. 85, 1528 (2000); K.S. Novoselov, A.K. Geim, S.V. Dubonos, E.W. Hill, I.V. Grigorieva, Nature (London) 426, 812 (2003); Y. Li, P. Xiong, and S. von Molnar, Appl. Phys. Lett. 80, 4644 (2002).

[3] V. Chandrasekhar, R.A. Webb, M.J. Brady, M.B. Ketchen, W.J. Gallagher, and A. Kleinsasser, Phys. Rev. Lett. 67, 3578 (1991); W. Wernsdorfer, K. Hasselbach, D. Mailly, B. Barbara, A. Benoit, L. Thomas and G. Suran, J. Magn. Magn. Mater. 145, 33 (1995).
[4] J.A. Sidles, J.L. Garbini, K.L. Bruland, D. Rugar, O. Züger, S. Hoen, and C.S. Yannoni, Rev. Mod. Phys. 67, 249 (1995).

[5] H.J. Mamin and D. Rugar, Appl. Phys. Lett. 79, 3358 (2001); B.C. Stipe, H.J. Mamin, C.S. Yannoni, T.D. Stowe, T.W. Kenny and D. Rugar, Phys. Rev. Lett. 87, 277602 (2001); H.J. Mamin, R. Budakian, B.W. Chui, D. Rugar, Phys. Rev. Lett. 91, 207604 (2003); G.P. Berman, F. Borgonovi, and V.I. Tsifrinovich, quant-ph/0306107

[6] D. Rugar, R. Budakian, H.J. Mamin and B.W. Chui, Nature 430, 329 (2004).

[7] S.A. Gurvitz, Phys. Rev. B 56, 15215 (1997); I.L. Aleiner, N.S. Wingreen, and Y. Meir, Phys. Rev. Lett. 79, 3740 (1997); Y. Levinson, Europhys. Lett. 39, 299 (1997); E. Buks, R. Schuster, M. Heiblum, D. Mahalu, and V. Umansky, Nature 391, 871 (1998).

[8] A. Shnirman and G. Schön, Phys. Rev. B 57, 15400 (1998); Y. Makhlin, G. Schön, and A. Shnirman, Phys. Rev. Lett. 85, 4578 (2000); Y. Makhlin, G. Schön, and A. Shnirman, Rev. Mod. Phys. 73, 357 (2001).

[9] M. Büttiker, Phys. Rev. Lett. 57, 1761 (1986). 
[10] X.Q. Li, W.K. Zhang, P. Cui, J.S. Shao, Z.S. Ma, and Y.J. Yan, Phys. Rev. B 69, 085315 (2004).

[11] J. Reijniers and F.M. Peeters, J. Appl. Phys. 87, 8088 (2000).

[12] K.S. Novoselov, S.V. Morozov, S.V. Dubonos, M. Missous, A.O. Volkov, D.A. Christian, and A.K. Geim, J. Appl. Phys. 93,10053 (2003).

[13] A. Oral and S.J. Bending, Appl. Phys. Lett. 69, 1324(1996). 